Bioelectrochemistry and Bioenergetics, 16 (1986) 565-567

A section of J. Electroanal. Chem., and constituting Vol. 212 (1986)

Elsevier Sequoia S.A., Lausanne - Printed in The Netherlands

\title{
971 - DIGRESSION ON BIOCHEMICAL MEMBRANE REACTIVITY IN WEAK ELECTROMAGNETIC FIELDS *
}

\section{EBERHARD NEUMANN}

Department of Physical and Biophysical Chemistry, Faculty of Chemistry, University of Bielefeld, P.O. Box 8640, D-4800 Bielefeld (F.R.G.)

(Manuscript received May 27th 1986)

An increasing number of studies suggests that biological organisms, cells and tissues are affected in growth, differentiation and proliferation by externally applied weak electromagnetic fields. The detailed mechanisms of the various field effects are not understood.

From a physico-chemical point of view it is still very puzzling that the observations refer to field strengths and field energies that hardly emerge out of the thermal noise. Clearly, the electric fields primarily act on charges, i.e. on free ions, on dipoles, on ionic and dipolar groups of larger molecules.

As far as small ions and dipoles are involved, it is well known that electric field strengths of $10-50 \mathrm{kV} \mathrm{cm}^{-1}$ are required to produce a few percent displacement of biochemical reaction systems [1]. Macromolecular protein structures and linear polyelectrolytes at low ionic strength exhibit conformational changes in the range of $1-10 \mathrm{kV} \mathrm{cm}^{-1}$.

Because ordinary cellular structures have no, or extremely small, permanent magnetic moments, directly measurable effects of magnetic fields on chemical reactivity are only expected in the magnetic field strength range of $\geqq 1$ Tesla. Here, too, it is unlikely that weak magnetic fields or the magnetic component of electromagnetic fields are operative at the level of small molecules and simple binding equilibria [2-6].

In the case of cellular effects of weak ( $\mathrm{mV}, \mathrm{V})$ electromagnetic fields it is very likely that they are of an indirect nature. In order to rationalize field effects by weak fields it is necessary to think of amplification units by which weak external voltages are transformed in the $\mathrm{kV} \mathrm{cm}^{-1}$ range. It appears that cell membranes may serve as such an amplification element [3].

\footnotetext{
* Summary of the invited lecture delivered at the VIIIth International Symposium on Bioelectrochemistry and Bioenergetics, Bologna, June 24th-29th 1985. The complete text was not sent.
} 
The primary process in the amplification cascade clearly is the accumulation of ions near the surfaces of the membranes exposed to the ionic environments of the cell exterior and interior, respectively. For spherical cells this is at the poles in the direction of the external electric field where the maximum interfacial charging of the membrane occurs. The time constant $\tau_{m}$ of the ionic surface charging depends on the size and ion content of the cell and its external medium. A typical value for a cell of radius $r=1 \mu \mathrm{m}$ is $\tau_{m} \approx 10 \mathrm{~ns}$.

The electric potential difference resulting from this interfacial charging is also dependent on the cell radius and, in addition, on the external electric field strength [3]. The field-induced potential difference is superimposed on the intrinsic natural membrane potential (difference).

It may be recalled that $\mathrm{mV}$ changes in the electric potential difference correspond to $\mathrm{kV} \mathrm{cm} \mathrm{cm}^{-1}$ changes in the electric field strength of a membrane of about $10 \mathrm{~nm}$ thickness. Therefore externally induced interfacial membrane charging causing electric potential changes of only a few $\mathrm{mV}$, may lead to appreciable alterations in the cross-membrane electric field strength. The field strength variation, in turn, will affect all binding equilibria involving ionic and dipolar and polarizable species. By introducing a characteristic Gibbs free energy function and a dielectrochemical potential [2], the electric field dependence of the distribution coefficients and equilibrium constants can be described quantitatively. In addition, the formalism demonstrates that the extent of the electric shift in chemical reactions is the larger the higher is the already existing membrane field.

As far as cross-membrane electrodiffusive processes are concerned, e.g. $\mathrm{Ca}^{2+}$ influx into the cell interior, it is a surprise that the external field may not affect primarily the electric part of the transport-driving force (electrochemical potential). Rather, the electrically induced ion accumulation increases the chemical concentration gradient so that a rather large increase in the transport rate is caused. For instance, if the interfacial charging causes a change in the natural electric membrane potential difference from $-70 \mathrm{mV}$ to $-77 \mathrm{mV}$, i.e. by $10 \%$, this corresponds to a change in the ratio of chemical activities by a factor of 1.3 or $30 \%$ (in the stationary state). Electrodiffusive $\mathrm{Ca}$ influx triggered by, say, hormone action will be increased by the same factor. Thus, external electric (and electromagnetic) stimulation may stimulate the transport of ions, ionic and dipolar metabolites primarily by the interfacial charging process. In this manner the cell membrane acts as a transducer and amplification element for small electromagnetic fields. We may view the membrane and its immediate environment (surface compartments [7]) as microreaction spaces that are particularly sensitive to external electric forces.

\section{REFERENCES}

1 E. Neumann, in Topics of Bioelectrochemistry and Bioenergetics, G. Milazzo (Editor), Wiley, New York, 1981, Vol. 4, pp. 113-160.

2 E. Neumann, in Modern Bioelectrochemistry, F. Gutmann and H. Keyzer (Editors), Plenum Press, New York, 1986, pp. 97-175. 
3 H.P. Schwan, in Advances in Biological and Medical Physics, Academic Press, New York, 1957, Vol. 5 , pp. 147-209.

4 H.P. Schwan, Neurosci. Res. Progr. Bull., 15 (1977) 88.

5 H.P. Schwan, in Advances in Biological Effects and Dosimetry of Low Energy Electromagnetic Fields, Plenum Press, New York, 1983.

6 M. Eigen and L.C.M. DeMaeyer, Tech. Org. Chem., 8 (1963) 895.

7 M. Blank and J.S. Britten, Bioelectrochem. Bioenerg., 5 (1978) 528. 